\title{
Effect of N-Terminal Myristoylation on the Active Conformation of $\mathrm{G} \alpha_{i 1}-\mathrm{GTP}$
}

Siri C. van Keulen ${ }^{\circledR}$ and Ursula Rothlisberger*

Institut des Sciences et Ingénierie Chimiques, École Polytechnique Fédérale de Lausanne (EPFL), CH-1015 Lausanne, Switzerland

Supporting Information

ABSTRACT: G proteins are part of the G-protein-coupled receptor (GPCR) signal transduction cascade in which they transfer a signal from the membrane-embedded GPCR to other proteins in the cell. In the case of the inhibitory G-protein heterotrimer, permanent $\mathrm{N}$-terminal myristoylation can transiently localize the $\mathrm{G} \alpha_{i}$ subunit at the membrane as well as crucially influence $\mathrm{G} \alpha_{i}$ 's function in the GTP-bound conformation. The attachment of lipids to proteins is known to be essential for membrane trafficking; however, our results suggest that lipidation is also important for proteinprotein interactions during signal transduction. Here we investigate the effect of myristoylation on the structure and dynamics of soluble $\mathrm{G} \alpha_{i 1}$ and its possible implication for signal transduction. A $2 \mu$ s classical molecular dynamics simulation of a myristoylated $\mathrm{G} \alpha_{i 1}-\mathrm{GTP}$ complex suggests that the myristoyl-induced conformational changes of the switch II and alpha helical domains create new possibilities for protein-protein interactions and emphasize the importance of permanent lipid attachment for the conformation and functional tunability of signaling proteins.

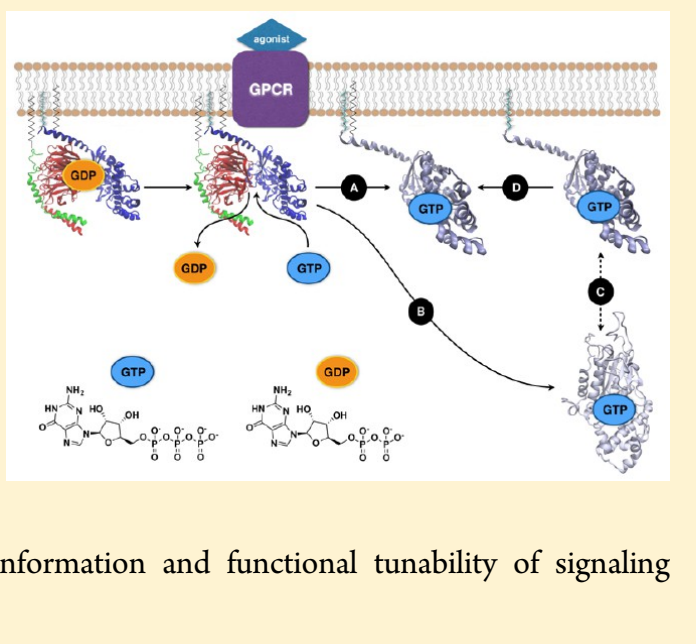

T -protein-coupled receptor (GPCR) pathways are of high interest since their signal transduction cascades play an important role in several diseases such as heart failure, hypertension, and obesity. The first proteins that will transfer an extracellular signal, received by a GPCR, to other regions in the cell, are $\mathrm{G}$ proteins, which are therefore crucial messengers in GPCR regulated signaling. $\mathrm{G}$ proteins are built from an $\alpha, \beta$, and $\gamma$ subunit of which the $\alpha$ subunit is able to interact with guanosine diphosphate (GDP) in the protein's inactive state or with guanosine-5' - triphosphate (GTP) in the active conformation. The interaction between a heterotrimeric $\mathrm{G}$ protein and an active GPCR induces GDP/GTP exchange in the G $\alpha$ subunit. $^{1-3}$ A concurrent destabilization of the interface between $\mathrm{G} \alpha$ and the $\mathrm{G} \beta \gamma$ dimer ${ }^{4}$ leads to soluble $\mathrm{G} \alpha$, thereby decreasing its concentration close to the membrane, in favor of the one in the cytosol. ${ }^{5}$ The specificity by which G-protein subunits interact with receptors and effectors defines the variety of responses that a cell is capable of providing due to an extracellular signal. Hence, understanding the molecular mechanism of $G$ proteins' binding to their cellular partners is a key step in understanding GPCR signaling cascades and can shed more light on the malfunctioning of GPCR signaling in humans.

Similarly to G proteins, smaller monomeric GTPases such as the ADP-ribosylation factor (ARF) proteins are also activated by GDP/GTP exchange. The localization of ARF proteins, like ARF1, at the membrane depends on whether the protein is interacting with a GDP or a GTP molecule. ${ }^{6,7}$ In addition, ARF1's location is not only determined by nucleotide interaction but also by lipidation of the $\mathrm{N}$-terminus through

myristoylation. The myristoyl group interacts with the membrane in ARF1's active conformation while it is interacting with a hydrophobic pocket on the protein in the inactive soluble state. Hence, GDP/GTP exchange and the myristoyl moiety are important for membrane localization and the solubility of ARF $1 .^{8}$ In the case of G proteins, similar factors can be found that influence the localization of $\mathrm{G} \alpha$ subunits. Besides GDP/GTP exchange, lipidation also plays a role in $\mathrm{G} \alpha$ localization. All $\mathrm{G} \alpha$ families ( $\mathrm{G} \alpha_{s}$ (stimulatory), $\mathrm{G} \alpha_{i}$ (inhibitory), $\mathrm{G} \alpha_{q}, \mathrm{G} \alpha_{12}$ ) can reversibly bind palmitoyl groups to the $\mathrm{N}$-terminus of their $\alpha$ subunit (except for G $\alpha_{t}$ from the $\mathrm{G} \alpha_{i}$ family), which anchors the subunit to the membrane, but only the $\mathrm{G} \alpha_{i}$ family has a myristoyl group attached to its $\mathrm{N}$-terminus in addition to the palmitoyl moiety. ${ }^{9,10}$ Unlike palmitoylation, myristoylation only enables transient binding to the membrane, which can lead to an equilibrium between a membrane-bound and a solvated state. ${ }^{11}$ Moreover, myristoylation is an irreversible process which results in myristoyl attachment throughout the lifetime of the protein. ${ }^{10}$ Hence, the increased solubility of all G $\alpha$ families' active $\mathrm{G} \alpha-\mathrm{GTP}$ complex is achieved by a combination of (i) dissociation of the G-protein heterotrimer, (ii) depalmitoylation, ${ }^{12,13}$ and (iii) detachment of the $\alpha$-subunit $\mathrm{N}$-terminal helix from the lipid bilayer. The solubility of the $\mathrm{G} \alpha_{i}$ family is also influenced by myristoylation

Received: April 25, 2016

Revised: November 13, 2016

Published: December 12, 2016 


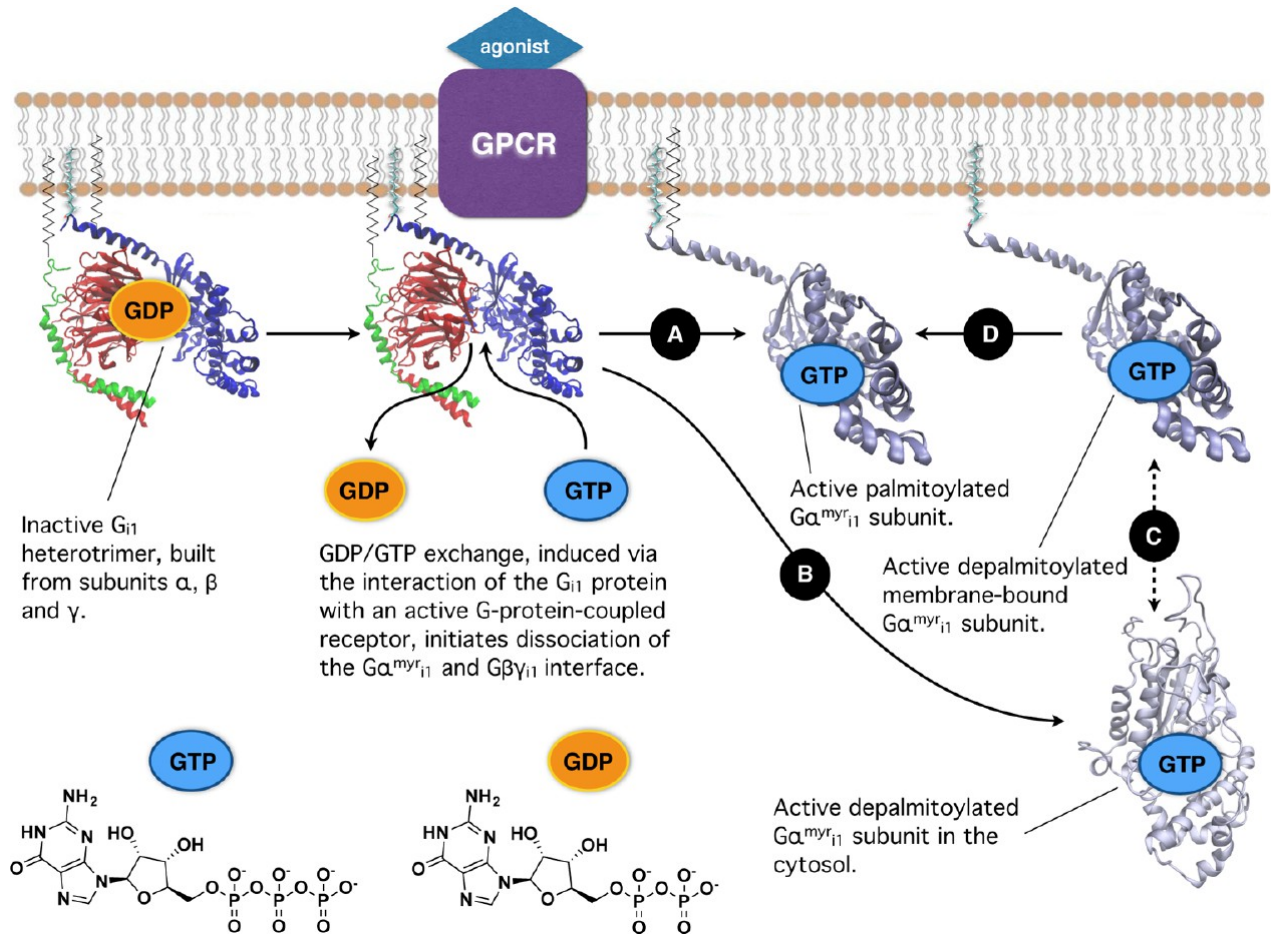

Figure 1. Proposed activation mechanism of $\mathrm{G}_{i 1}^{\mathrm{myr}}$ in which an activated GPCR induces GDP/GTP exchange that leads to dissociation of the G $\alpha_{i 1}$ and $\mathrm{G} \beta \gamma$ interface. (A) represents the activation of $\mathrm{G} \alpha_{i 1}^{\text {myr }}$ in which the $\mathrm{N}$-terminal palmitoyl group is still present. (B) shows the conversion to activated depalmitoylated $\mathrm{G} \alpha_{i 1}^{\text {myr }}$ which results in an equilibrium $(\mathrm{C})$ between a solvated $\mathrm{G} \alpha_{i 1}^{\text {myr }}-\mathrm{GTP}$ and a membrane-bound $\mathrm{G} \alpha_{i 1}^{\mathrm{myr}}-\mathrm{GTP}$ complex. (D) represents the palmitoylation of active membrane-bound $\mathrm{G} \alpha_{i 1}^{\text {myr }}$.

as the post translational modification induces an equilibrium between membrane-bound and cytosolic forms. ${ }^{14}$

Besides the solubility, also the conformation of active $\mathrm{G} \alpha_{i 1}$ has been shown to be affected by myristoylation, ${ }^{15}$ as the soluble non-myristoylated $\mathrm{G} \alpha_{i 1}-\mathrm{GTP}$ complex does not maintain the secondary structure of its $\mathrm{N}$-terminal helix, while the active soluble myristoylated $\mathrm{G} \alpha_{i 1}$ subunit shows a more structured $\mathrm{N}$-terminal tail and is able to position the $\mathrm{N}$ terminus close to the $\mathrm{G} \alpha_{i 1}$ subunit. $^{15}$ The importance of permanent $\mathrm{N}$-terminal myristoylation of the $\mathrm{G} \alpha_{i 1}$ subunit is not only shown by its influence on $\mathrm{G} \alpha_{i 1}-$ GTP's structure but also by the dependence of the function of the $\alpha$ subunit on the presence of the myristoyl moiety. ${ }^{16,17}$ In the case of adenylyl cyclase type 5 (AC5), for example, myristoylated $\mathrm{G} \alpha_{i 1}\left(\mathrm{G} \alpha_{i 1}^{\text {myr }}\right)$ can interact with the catalytic domain of AC5 and inhibit its catalytic function. However, a non-myristoylated $\mathrm{G} \alpha_{i 1}\left(\mathrm{G} \alpha_{i 1}^{\text {non }}\right)$ subunit is incapable of interacting with $\mathrm{AC}$ and can therefore not inhibit AC's ATP conversion pathway. ${ }^{16}$ For the stimulatory $\mathrm{G} \alpha_{s}$ though, the presence of the $\mathrm{N}$-terminus is not crucial for its interaction with $\mathrm{AC}$ as is demonstrated by the obtained X-ray structure of Tesmer et al. ${ }^{18}$ It can be proposed that in the active $\mathrm{G} \alpha_{s}$ state, the $\mathrm{N}$-terminus is less structured, similar to the results of Preininger et al. for the nonmyristoylated $\mathrm{G} \alpha_{i 1}-\mathrm{GTP}$ complex, which could suggest that the overall active $\mathrm{G} \alpha_{s}$ conformation is not significantly altered by the structure of the $\mathrm{N}$-terminal helix. ${ }^{15}$

Although myristoylation appears to be an important difference between the stimulating $\mathrm{G} \alpha_{s}$ and inhibiting $\mathrm{G} \alpha_{i}$ families and is furthermore crucial for $\mathrm{G} \alpha_{i 1}$ 's conformation, no X-ray structures of myristoylated $\mathrm{G} \alpha_{i}$ proteins are currently available in the Protein Data Bank (PDB). ${ }^{19}$ The available Xray structures of active $\mathrm{G} \alpha_{s}$ and $\mathrm{G} \alpha_{i 1}^{\text {non }}$ are similar as is shown via a root-mean-square deviation (RMSD) of $1.07 \AA$ between the two structures (Supporting Figure 1). Especially around the proposed protein interaction site, the switch II region, the subunits align well, while their function is inverse in the cytoplasmatic domain. Therefore, it is unclear to what extent the available $\mathrm{G} \alpha_{i 1}^{\text {non }}$ structures are representative of the complete conformational range of the active $\mathrm{G} \alpha_{i 1}$ protein. Hence, understanding the effects of myristoylation on $\mathrm{G} \alpha_{i 1}$ can possibly help rationalize the impact on $\mathrm{G} \alpha_{i 1}$ 's conformation and the difference in biological function between $\mathrm{G} \alpha_{s}$ and $\mathrm{G} \alpha_{i 1}$. Additionally, it can contribute to a better understanding of Gprotein activation and provide a new perspective on the role of lipid attachment in protein function.

To shed light on this issue, we have studied $\mathrm{G} \alpha_{i 1}^{\mathrm{myr}}$ in its active state using classical molecular dynamics (MD) simulations of a $\mathrm{G} \alpha_{i 1}^{\text {myr }}$ model (Figure 3) based on $\mathrm{G} \alpha_{i 1}^{\text {non }} \mathrm{X}$ ray structures and an ARF1 protein NMR structure (Figure 2 and section Homology Modeling). A second simulation was performed as well with the same $\mathrm{G} \alpha_{i 1}-\mathrm{GTP}$ model in the absence of its myristoylated $\mathrm{N}$-terminus as a cross validation.

A $2 \mu \mathrm{s}$ simulation of the $\mathrm{G} \alpha_{i 1}^{\mathrm{myr}}-\mathrm{GTP}$ complex identifies a stable binding site for the myristoyl group in between $\alpha 1, \alpha 5$, and $\beta 2-\beta 3$, and demonstrates the effect of myristoyl binding for important interaction sites such as the switch II region and the nucleotide binding pocket. Significant changes occur in the conformation of the switch II region upon incorporation of the myristoyl moiety. Moreover, a large rearrangement of $\alpha \mathrm{B}$ in the alpha helical (AH) domain of the $\alpha$ subunit takes place. These changes in the secondary structure of the switch II region and the AH domain could have an important effect on the way the $\mathrm{G} \alpha_{i 1}^{\mathrm{myr}}$ subunit is able to interact with proteins in the cytosol. The proposed mechanism for $\mathrm{G}_{i 1}^{\mathrm{myr}}$ activation is described in Figure 1. 

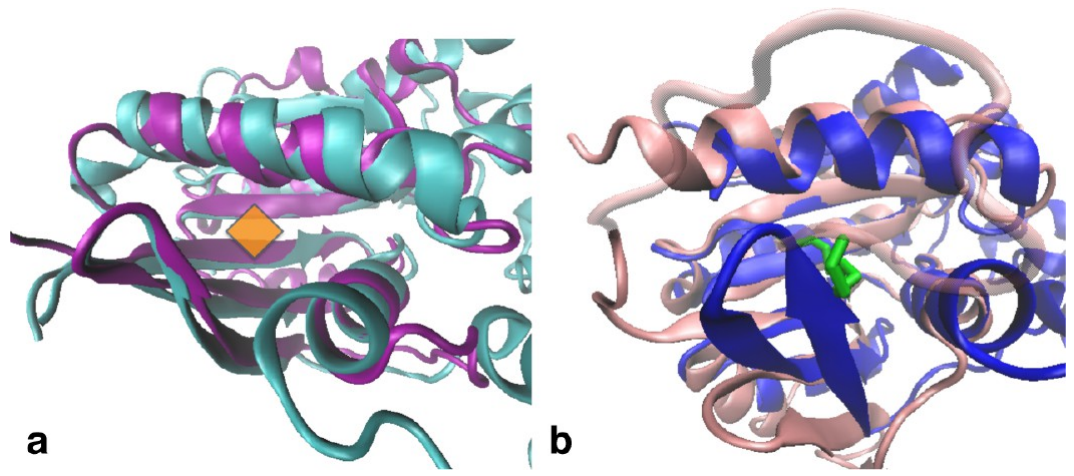

Figure 2. Comparison of ARF1 and $\mathrm{G} \alpha_{i 1}$. (a) Alignment of the $\mathrm{C} \alpha$ atoms of the ARF1-GTP complex in purple (PDB code $\left.2 \mathrm{KSQ}\right)$ and the G $\alpha_{i 1}^{\text {non }}-$ GDP conformation in cyan (PDB code 1AS3) zoomed in on ARF1's myristoyl binding site (orange diamond) in the inactive conformation. (b) Alignment of the C $\alpha$ atoms of the ARF1-GDP complex in pink (PDB code $2 \mathrm{~K} 5 \mathrm{U}$ ) and the G $\alpha_{i 1}^{\text {non }}-$ GTP conformation in blue (PDB code 1GIA) zoomed in on ARF1's myristoyl binding site. The myristoyl moiety of ARF1 is depicted in green and is interacting with a hydrophobic pocket, located on ARF1.

\section{METHODS}

Homology Modeling. The refinement protocol automodel from Modeler $9.14^{20,21}$ was employed to obtain the initial homology model of the myristoylated Rattus norvegicus $\mathrm{G} \alpha_{i 1}$ subunit (UniprotKB P10824) that was used to start the classical $\mathrm{MD}$ runs (Figure 3a and Supporting Figure 2). In total 100 structures were obtained with Modeler 9.14. Besides maintaining the secondary structure of the main template, $4 \mathrm{PAQ}$ and avoiding bad contacts within the structure, the selection of the initial model for simulation was based on (I) the presence of important hydrogen bonds between GTP and the active site that are shown in the X-ray structure, (II) maintaining the secondary structure present in the included templates of the regions that were not part of the main template, PDB code $4 \mathrm{PAQ}$ (III) the position of the myristoyl moiety that had to be located within $4 \AA$ of the hydrophobic pocket as an initial guess for the moiety's location.

A combination of three different X-ray structures (PDB codes: $2 \mathrm{~K} 5 \mathrm{U}, 1 \mathrm{AS} 3$ and $4 \mathrm{PAQ}$ ) was incorporated in the initial homology model. ${ }^{6,22,23} 4 \mathrm{PAQ}$ a Rattus norvegicus $\mathrm{G} \alpha_{i 1}^{\text {non }}-5^{\prime}$ guanosine-diphosphate-monothiophosphate $(\mathrm{GTP} \gamma \mathrm{S})$ complex, was used as a main template. GTP $\gamma \mathrm{S}$ is an analogue of GTP, which is used in the crystallization process of the $\mathrm{G} \alpha_{i 1}$ subunit as GTP $\gamma \mathrm{S}$ is not able to undergo the hydrolysis mechanism in the active site of the protein. 1AS3, a $R$ norvegicus $\mathrm{G} \alpha_{i 1}^{\text {non }}-$ GDP conformation, covered the $\mathrm{N}$ (residues 9-34) and the $\mathrm{C}$ (residues 328-354) terminus of the $\mathrm{G} \alpha_{i 1}$ subunit. In order to obtain an initial guess for the location of the myristoyl moiety, a myristoyl conformation of ARF1 from Saccharomyces cerevisiae (UniprotKB P11076) with PDB code 2K5U was used (myristoyl group and residues 1-7). The 2K5U NMR structure (Figure 2) was also employed to model the interswitch toggle (Results and Discussion and Supporting Methods), residues 179-220 of $\mathrm{G} \alpha_{i 1}$, which enables communication between the myristoyl and the nucleotide binding site. ${ }^{24}$

The location of GTP $\gamma$ S and the $\mathrm{Mg}^{2+}$ ion was taken from 4PAQ in order to place the ion and guide the GTP molecule in the nucleotide binding site. Also water molecules (16 molecules in total) surrounding GTP $\gamma \mathrm{S}$ in $4 \mathrm{PAQ}$ were incorporated in the model. Since different structures were used to obtain the homology model, restraints were employed for the construction of the initial model to maintain important hydrogen bonds in the nucleotide binding site and to position the myristoyl moiety close to the hydrophobic pocket (Supporting Figure 2).
Classical Molecular Dynamics Simulations. The $\mathrm{G} \alpha_{i 1}$ subunit together with its bound myristoyl group, $\mathrm{Mg}^{2+}$ ion, and GTP was simulated for $2 \mu \mathrm{s}$ at $310 \mathrm{~K}$ and 1 bar using a NoséHoover thermostat and an isotropic Parrinello-Rahman barostat. Additionally, about 42000 water molecules and 150 $\mathrm{mM} \mathrm{KCl}$ are present in the simulated system. The force fields used for the protein and the water molecules are AMBER99SB ${ }^{25}$ and TIP3P, ${ }^{26}$ which were employed by Gromacs $4.66^{27,28}$ to perform the runs. For GTP, the force field parameters generated by Meagher et al. were used. ${ }^{29}$ The adjusted force field parameters for $\mathrm{K}^{+}$and $\mathrm{Cl}^{-}$were taken from Joung et $\mathrm{al}^{30}{ }^{30} \mathrm{The} \mathrm{Mg}^{2+}$ parameters originated from Allnér et al. ${ }^{31}$ The charges for the myristoyl group were obtained with Gaussian $09^{32}$ based on Hartree-Fock calculations in combination with a 6-31G* basis set and using the AMBER RESP procedure $^{33}$ (Supporting Table 1). Appropriate atom types from the AMBER99SB force field were selected to complete the myristoyl description.

Electrostatic interactions were calculated with the Ewald particle mesh method with a real space cutoff of $12 \AA$. Bonds involving hydrogen atoms were constrained using the LINCS algorithm. The time integration step was set to $2 \mathrm{fs}$.

Calculation of Electrostatic Surfaces. The electrostatic surface of several $\mathrm{G} \alpha_{i 1}$ subunits was calculated via APBS 1.3. ${ }^{34,35}$ The multigrid method together with the monopole boundary conditions and spline-based discretization of the delta functions was used. The ion concentration was set to $150 \mathrm{mM}$ at a temperature of $310 \mathrm{~K}$. The default settings for the biomolecular (2.00) and solvent (78.54) dielectric constants were used. PDB2PQR 2.0.0 $0^{35,36}$ was employed to perform the preprocessing of the conformations with the AMBER force field in order to calculate the atomic radii, charges and position of the hydrogen atoms.

Structure Superpositions and Images. Multiprot ${ }^{37}$ and $\mathrm{VMD}^{38}$ were used to align protein structures. The Align tool in Uniprot $^{39}$ was used to align protein sequences. Images were prepared with VMD. ${ }^{38}$

\section{RESULTS AND DISCUSSION}

Classical Molecular Dynamics Simulations of $\mathrm{G} \alpha_{i 1}^{\mathrm{myr}}$. During the $2 \mu$ s of classical MD simulation, the effects of the myristoyl group on the structure of the active $\mathrm{G} \alpha_{i 1}$ subunit become apparent (Figure 3 and Supporting Figure 4). In the equilibrated conformation, the covalently bound myristoyl 

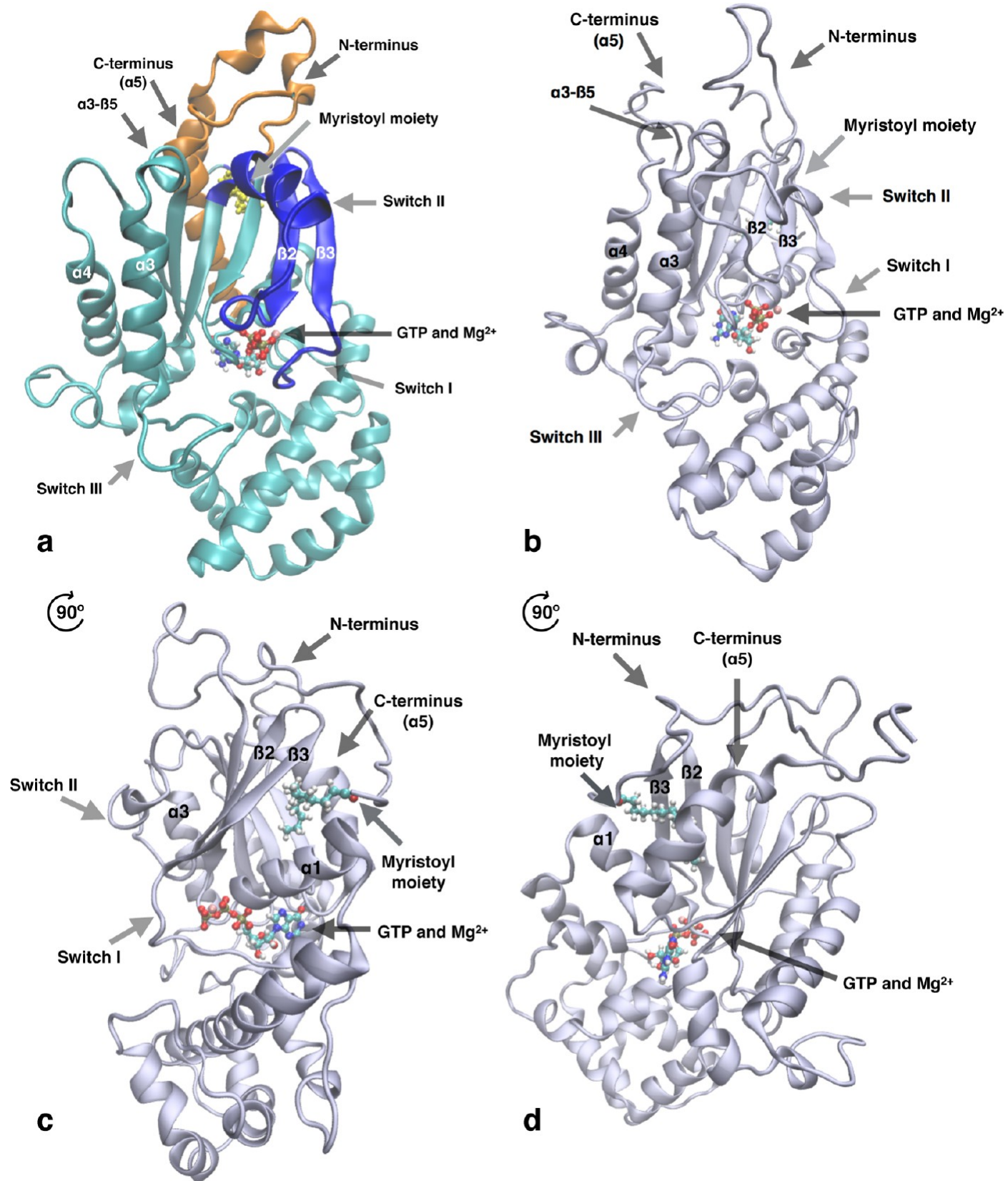

Figure 3. Structural overview of $\mathrm{G} \alpha_{i 1}^{\mathrm{myr}}-\mathrm{GTP}$ and comparison with X-ray structures. (a) Representation of the initial homology model that was used to start the classical MD simulation. The template for the cyan region was PDB entry 4PAQ for the orange area the PDB structure $1 \mathrm{AS} 3$ was used and the blue part was modeled after PDB entry $2 \mathrm{~K} 5 \mathrm{U}$. The myristoyl moiety is depicted in yellow. (b, c, d) Three different views of the final G $\alpha_{i 1}^{\text {myr }}$ structure in which the myristoyl moiety, GTP, and $\mathrm{Mg}^{2+}$ are also shown.

group interacts with the $\mathrm{G} \alpha_{i 1}$ protein via $\beta 2-\beta 3$, the $\alpha 5$ helix and the $\alpha 1$ helix (Figure $3 \mathrm{~b}-\mathrm{d}$ ). One direct effect of the presence of the myristoyl group is an outward conformation of $\beta 2-\beta 3$ in order to provide space for the lipid moiety (Figure $4 a)$. The $\beta 2-\beta 3$ strands are shifted down by two residue units (Figure $4 \mathrm{~b}$ ), which increases the number of residues that are part of the switch II region. This shift of two residue units has also been observed in myristoyl containing ARF proteins and is called the interswitch toggle. ${ }^{24}$ This is not too surprising since ARF's signature sequence for the interswitch toggle, wDvGGqxxxRxxW, is also present in $\mathrm{G} \alpha_{i 1}$ 's sequence in the region that includes $\beta 3$ and the switch II region (Figure 5). ${ }^{24}$ The interswitch toggle facilitates communication within the $\mathrm{G} \alpha_{i 1}$ subunit between the nucleotide binding site and the myristoyl moiety. ${ }^{24}$ The effect on the nucleotide binding site (Supporting Results and Discussion) is that since there are more residues available from the switch II region, the $\mathrm{N}$ terminal region of the switch II region is able to interact with the $\alpha 3$ helix, which stabilizes the structure of the switch II region (Figure 4c). A cross-validation simulation was also performed in which the $\mathrm{N}$-terminus was cut off until the 31th residue, $\mathrm{G} \alpha_{i 1}^{\text {non }}-\mathrm{GTP}$, to monitor the effect on the initial model (Supporting Figure 5). The result showed indeed a hydrophobic pocket that was closed by $\beta 2-\beta 3$ and a switch II region that returned to the conformation of the switch II region of the $\mathrm{G} \alpha_{i 1}^{\text {non }}-\mathrm{GTP} \gamma \mathrm{S}$ X-ray structure (PDB code: 1GIA). The upward movement of the interswitch toggle and the full rearrangement of the nucleotide binding site was not observed in the $1 \mu \mathrm{s}$ 

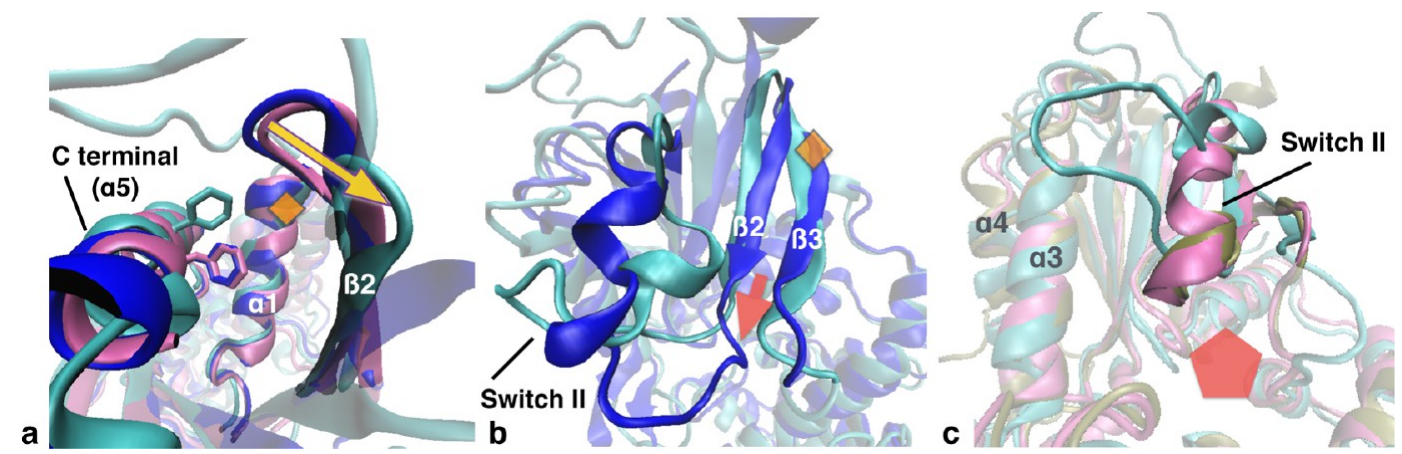

Figure 4. (a) Zoom on the proposed myristoyl binding site of aligned $\mathrm{G} \alpha_{i 1}$ subunits interacting with GTP, a GTP analogue or GDP. The mauvecolored structure is a G $\alpha_{i 1}^{\text {non }}-$ GTP $\gamma S$ complex (PDB code 1GIA), and the model, G $\alpha_{i 1}^{\text {myr }}-$ GTP, after about $\sim 1.8 \mu$ s is shown in cyan. The G $G_{i 1}^{\text {non }}-$ GDP conformation (PDB code 1GP2) is colored blue. The orange diamond shows where the myristoyl group is connected to the G $\alpha_{i 1}$ subunit of the model. The yellow arrow specifies the outward movement of the $\beta 2-\beta 3$ domain upon myristoyl binding. Additionally, Phe336's orientation is shown for all three structures in the previously described structure colors. (b) The $\beta 2-\beta 3$ shift between the $G_{i 1}^{\text {non }}-$ GDP and the G $\alpha_{i 1}^{\text {myr }}-G T P$ complexes is shown by the red arrow and the myristoyl binding site is depicted as an orange diamond. The color scheme for the structures is the same as in the (a) image. (c) Alignment of $\mathrm{G} \alpha_{i 1}$ subunits in which the difference in the switch II region is shown. The red pentagon is showing the location of the guanine nucleotide binding site. The color scheme of the $\mathrm{G} \alpha_{i 1}$ structures is the same as in the (a) image, except for the fact that the tan structure is the X-ray conformation of the G $\alpha_{s}-\mathrm{GTP} \gamma \mathrm{S}$ complex (PDB code $1 \mathrm{AZT}$ ). ${ }^{40}$

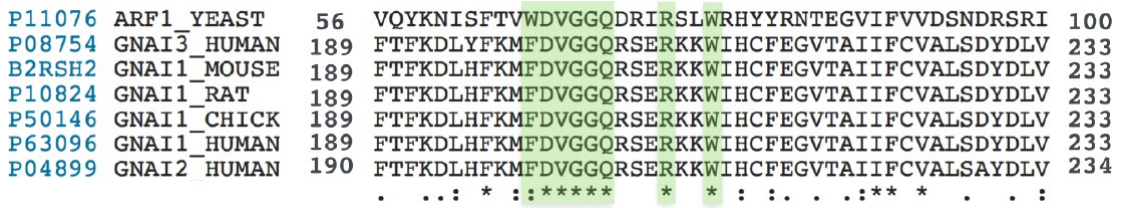

Figure 5. Alignment of several G $\alpha$ sequences and ARF1 from Saccharomyces cerevisiae.

classical MD simulation, which could be due to the limited length of the simulation. Overall, the structural rearrangements of the simulated $\mathrm{G} \alpha_{i 1}^{\mathrm{myr}}$ as a result of myristoyl binding, show that the effect of the myristoyl moiety on the conformation of the $\mathrm{G} \alpha_{i 1}^{\mathrm{myr}}$ subunit is not limited to the hydrophobic binding pocket but influences also other parts of the protein.

The change in conformation of $\mathrm{G} \alpha_{i 1}^{\mathrm{myr}}-\mathrm{GTP}$ is likely to also have implications for the interaction with other proteins, which could affect the overall function of $\mathrm{G} \alpha_{i 1}^{\mathrm{myr}}$ in the cytosol. In order to address the role of the altered conformation and to identify if it has an impact on protein-protein interactions in which active $\mathrm{G} \alpha_{i 1}$ is involved, the equilibrated model was compared to X-ray structures of $\mathrm{G} \alpha_{i 1}^{\text {non }}$ and $\mathrm{G} \alpha_{s}$. First, the overall changes in conformation are compared, and a mechanism for $\mathrm{G} \alpha_{i 1}^{\text {myr }}$ localization in the cytosol is proposed. Moreover, the important regions for myristoyl binding, GTP binding (Supporting Results and Discussion), and proteinprotein interactions are discussed to obtain a better understanding of the effect of permanent lipid attachment.

Two Conformations of Active $\mathbf{G} \alpha_{i 1}^{\text {myr }}$. When comparing the simulated model with the crystal structure of the $\mathrm{G} \alpha \alpha_{i 1}^{\text {non }}-$ GTP $\gamma$ S complex (PDB code 1GIA) ${ }^{41}$ in which the $\mathrm{N}$-terminus is not resolved, it becomes evident that in the myristoylated $\mathrm{G} \alpha_{i 1}-\mathrm{GTP}$ complex an outward orientation of $\beta 2-\beta 3$ is stabilized by the myristoyl in the hydrophobic pocket. Because of this change in conformation also the conformation of the switch II region is modified. In the X-ray structure of $\mathrm{G} \alpha_{i 1}^{\text {non }}-$ $\mathrm{GTP} \gamma \mathrm{S}$, however, the conformation of the complex is strikingly identical to the active $\mathrm{G} \alpha_{s}-\mathrm{GTP} \gamma \mathrm{S}$ conformation as the myristoyl binding pocket remains closed (Figure $4 \mathrm{c}$ and Supporting Figure 1). ${ }^{40,41}$ Although in both X-ray structures, $\mathrm{G} \alpha_{s}-\mathrm{GTP} \gamma \mathrm{S}$ and $\mathrm{G} \alpha_{i 1}^{\text {non }}-\mathrm{GTP} \gamma \mathrm{S}$, the N-terminus is not present, the $\mathrm{N}$-terminus of $\mathrm{G} \alpha_{s}-\mathrm{GTP}$ is suggested to be less structured in solution as it is not anchored on the protein by a myristoyl group or by the membrane, while in the case of $\mathrm{G} \alpha_{i 1}^{\mathrm{myr}}-\mathrm{GTP}$, the N-terminus adopts a more structured conformation in close proximity to the protein. ${ }^{15}$ This difference in myristoyl presence could suggest that the impact of the unresolved $\mathrm{N}$-terminus is less severe for the conformation of the protein in the case of $\mathrm{G} \alpha_{s}-\mathrm{GTP} \gamma \mathrm{S}$ than the one of $\mathrm{G} \alpha_{i 1}-\mathrm{GTP} \gamma \mathrm{S}$. $^{15}$

The X-ray structure of activated $\mathrm{G} \alpha_{i 1}^{\text {non }}$ and the simulated active $\mathrm{G} \alpha_{i 1}^{\mathrm{myr}}$ appear to represent two different conformations of $\mathrm{G} \alpha_{i 1}$ in the active state. The main difference between the two structures is the absence or presence of the myristoylated $\mathrm{N}$ terminus. Possibly, both conformations are able to exist under two different circumstances: one in which the myristoyl group is interacting with the protein's hydrophobic binding pocket and one in which it is immersed into the membrane, localizing $\mathrm{G} \alpha_{i 1}$ in close proximity to the membrane surface. This suggestion is consistent with experimental findings in which both membrane-bound and solvated conformations are simultaneously observed for active $\mathrm{G} \alpha_{i 1}^{\mathrm{myr}}{ }^{14}$ Consequently, in this view two forms of activated depalmitoylated $\mathrm{G} \alpha_{i 1}^{\mathrm{myr}}$ are present in equilibrium in the intracellular domain: the membrane-bound and the solvated form of $\mathrm{G} \alpha_{i 1}^{\mathrm{myr}}-\mathrm{GTP}$ (Figure 1). This also proposes that palmitoylation in combination with myristoylation could be responsible for different conformations of the $\mathrm{G} \alpha_{i 1}-\mathrm{GTP}$ complex by transiently or permanently binding the subunit to the lipid bilayer. This directionality induced by lipid attachment could supposedly result in selectivity for particular proteins, depending on $\mathrm{G} \alpha_{i 1}^{\mathrm{myr}}$ 's location in the cell.

The X-ray structure of $\mathrm{G} \alpha_{i 1}^{\text {non }}-\mathrm{GTP} \gamma \mathrm{S}$ is likely to represent the membrane-bound-like conformation of active $\mathrm{G} \alpha_{i 1}^{\mathrm{myr}}$ as the hydrophobic pocket for the myristoyl moiety, that is identified 


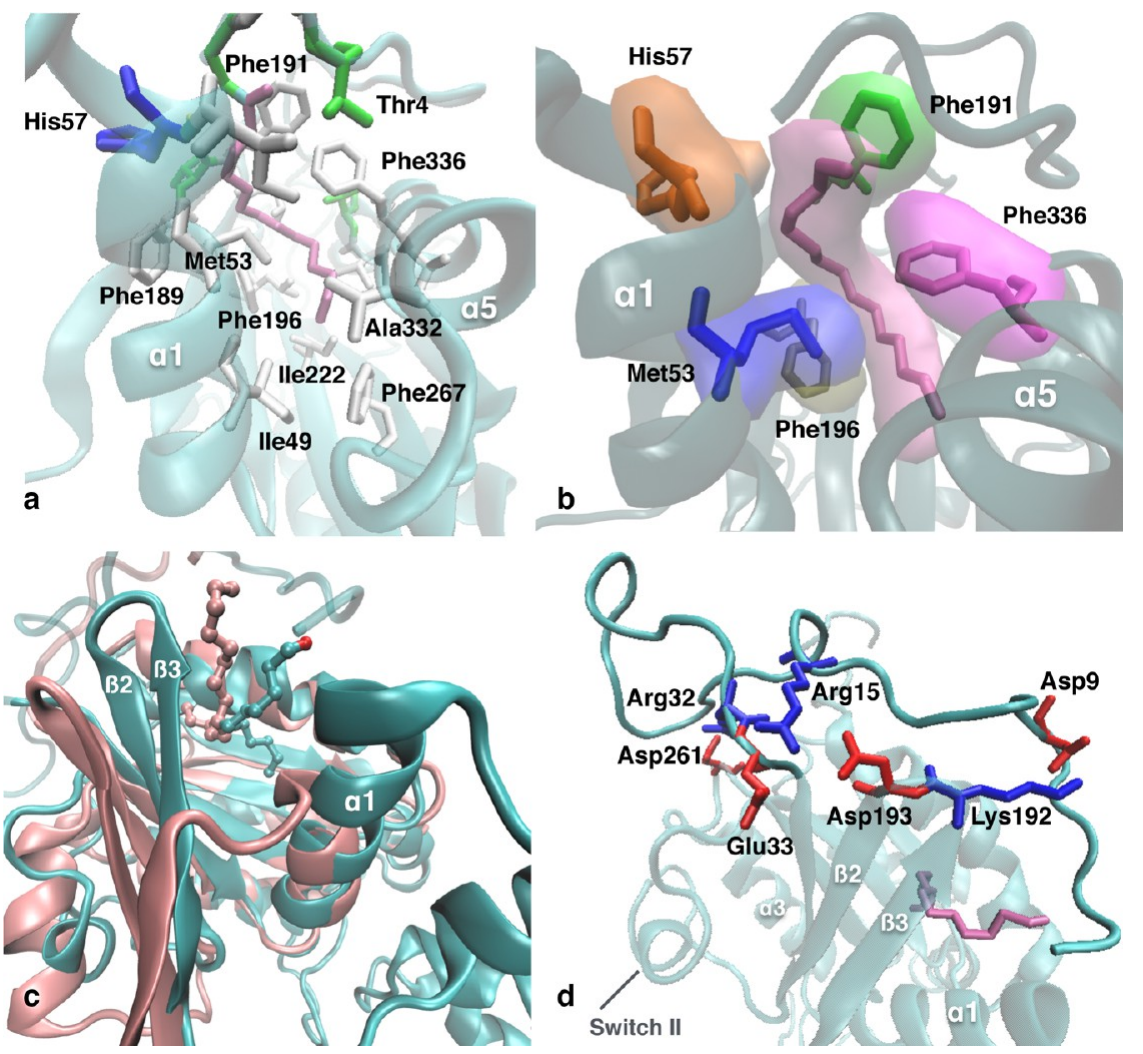

Figure 6. Detailed representation of $\mathrm{G} \alpha_{i 1}^{\text {myr }}$ s myristoyl binding site and $\mathrm{N}$-terminus. (a) Zoom on $\mathrm{G} \alpha_{i 1}^{\text {myr }}$ s hydrophobic binding pocket in which the myristoyl group (pink) is binding. Nonpolar residues are colored in white, polar residues are shown in green. (b) Myristoyl binding pocket of the simulated $\mathrm{G} \alpha_{i 1}^{\text {myr }}-$ GTP complex in which the myristoyl moiety (pink) and several residues are shown: Met53 (blue), His57 (orange), Phe189 (green), Phe191 (gray) and Phe336 (purple). In addition, the molecular volume sampled over a period of $500 \mathrm{~ns}$, from 1.6 to $2.1 \mu \mathrm{s}$, is shown for all described moieties. (c) Alignment of $\mathrm{G} \alpha_{i 1}^{\text {myr }}-$ GTP in the equilibrated conformation (cyan) at $\sim 1.8 \mu$ s of classical MD and ARF1-GDP (pink), PDB code $2 \mathrm{~K} 5 \mathrm{U}$. Besides the protein, also the location of the myristoyl group, GTP, GDP and $\mathrm{Mg}^{2+}$ are shown for both conformations. (d) Location of the N-terminus with respect to the rest of the $\mathrm{G} \alpha_{i 1}^{\mathrm{myr}}$ subunit. The myristoyl moiety is shown in pink. Negatively (red) and positively (blue) charged residues are shown on and around the N-terminus that keep the $\mathrm{N}$-terminal tail into place on the $\mathrm{G} \alpha$ subunit.

by the classical MD simulation to be between $\beta 2-\beta 3$, the $\alpha 5$ helix and the $\alpha 1$ helix, is unavailable. Consequently, the lipid will have to be located in a different hydrophobic site, most probably at the membrane itself. ${ }^{42}$ It is known from experiments that the myristoyl group alone is not enough to locate $\mathrm{G} \alpha_{i 1}$ at the membrane permanently but enables a transient stability which results in a fraction of the protein being in the membrane-bound state and another fraction in the solvated state. ${ }^{14}$ In fact, in order to stabilize its location on the membrane in a definitive manner, $\mathrm{G} \alpha_{i 1}^{\text {myr }}$ has to be palmitoylated, which results in a firm anchoring of the protein to the lipid bilayer. ${ }^{9,14,43}$ In view of this, the active conformation of $\mathrm{G} \alpha_{i 1}^{\mathrm{myr}}$ observed in the simulations appears to be a structural representative of solvated depalmitoylated $\mathrm{G} \alpha_{i 1}^{\mathrm{myr}}-\mathrm{GTP}$ with the myristoyl group packed away in a hydrophobic protein pocket (section Myristoyl Binding Site).

In the next sections, the $\mathrm{G} \alpha_{i 1}^{\mathrm{myr}}-\mathrm{GTP}$ structure in the solvated state will be described in more detail and compared to the $\mathrm{G} \alpha_{i 1}^{\text {non }}-\mathrm{GTP} \gamma \mathrm{S}$ conformation.

Myristoyl Binding Site. Conformational changes induced by the myristoyl moiety start with the modification of $\mathrm{G} \alpha_{i 1}^{m y r}$ s hydrophobic pocket (Figure 6a,b). Within the time scale of the $2 \mu \mathrm{s}$ simulation, the hydrophobic site in the $\mathrm{G} \alpha_{i 1}^{\mathrm{myr}}-\mathrm{GTP}$ complex assumes a more stable conformation. $\beta 2-\beta 3$ gets closer to $\alpha 1$ and the myristoyl tail reorients (Supporting Figure 6) while the region of the hydrophobic pocket remains similar to the ARF1-GDP complex (Figure 6c). The location of the myristoyl moiety in the pocket is close to the position of the myristoyl group in ARF1, but the orientation of the myristoyl tail differs between $\mathrm{G} \alpha_{i 1}^{\mathrm{myr}}-\mathrm{GTP}$ and ARF1-GDP. In the ARF1-GDP conformation the majority of the myristoyl is positioned between $\beta 2-\beta 3$ and $\alpha 5$, while the tail is pointing toward the C-terminal end of $\alpha 5$. In the $\mathrm{G} \alpha_{i 1}^{\text {myr }}-\mathrm{GTP}$ system, the myristoyl group is in between $\beta 2-\beta 3$ and $\alpha 5$ as well, but the tail is oriented toward $\alpha 1$ (Figure 6c). These alterations are not surprising in view of the fact that the changes in the hydrophobic pocket conformation could originate from the difference in the Ras domain between the two proteins. $\mathrm{G} \alpha_{i 1}$, for instance, has a longer $\alpha 1$ helix than ARF1, that can stabilize $\beta 2-\beta 3$. Additionally, an alpha helical domain is located between $\alpha 1$ and switch I in the $\mathrm{G} \alpha_{i 1}$ protein, which restrains the conformational flexibility of switch I in comparison to ARF1.

Several features of the hydrophobic pocket of $\mathrm{G} \alpha \alpha_{i 1}^{\mathrm{myr}}-\mathrm{GTP}$ can be rationalized through comparison with experimental findings (Supporting Results and Discussion). For instance, due to the location of the myristoyl group, an outward motion of Phe336 occurs during the classical MD simulation (Figure 4a), which has been linked to activation of the $\mathrm{G} \alpha_{i 1}$ subunit $^{22}$ (Supporting Results and Discussion).The identified hydrophobic pocket also opens up a new region for the investigation of myristoyl binding to $\mathrm{G} \alpha_{i 1}$. The presented data show that residues Met53 and Phe196, for instance, are in close proximity to the myristoyl group and therefore these residues could be candidates for mutagenesis studies as mutating these residues 
into a bulky hydrophobic residue, such as a tryptophan, could prohibit the myristoyl moiety from entering the hydrophobic pocket. Via these mutations a change in the conformation of the $\mathrm{G} \alpha_{i 1}$ subunit could be initiated, resulting in a hydrophobic pocket that is unavailable for the myristoyl moiety. This mutated $\alpha$ subunit would be myristoylated, however, it would be unable to interact with adenylyl cyclase type 5 due to the change in the hydrophobic pocket.

Besides the hydrophobic pocket, the conformation of the $\mathrm{N}$ terminus is also adjusted by the presence of the myristoyl group (Supporting Results and Discussion), which becomes anchored through the embedding of the myristoyl moiety in the hydrophobic binding pocket. In the $\mathrm{G} \alpha_{i 1}^{\mathrm{myr}}-\mathrm{GTP}$ complex, the $\mathrm{N}$-terminus is closely packed to the rest of the protein and is not only able to interact with $\beta 2-\beta 3$ and $\alpha 3-\beta 5$, stabilizing the conformation of the hydrophobic pocket, but can also form interactions with the C-terminal part of the switch II region (Figure 6d). The presence of the myristoyl moiety anchors the $\mathrm{N}$-terminus in a more rigid conformation that enables further stabilization of the hydrophobic pocket.

Switch II Region and Alpha Helical Domain. Available $\mathrm{X}$-ray structures and the data from the classical MD simulations propose at least two possible locations of the myristoyl moiety during different states of the $\mathrm{G} \alpha_{i 1}$ subunit (Table 1). The

\section{Table 1. Comparison of Different $\mathbf{G} \alpha_{i 1}$ States $^{a}$}

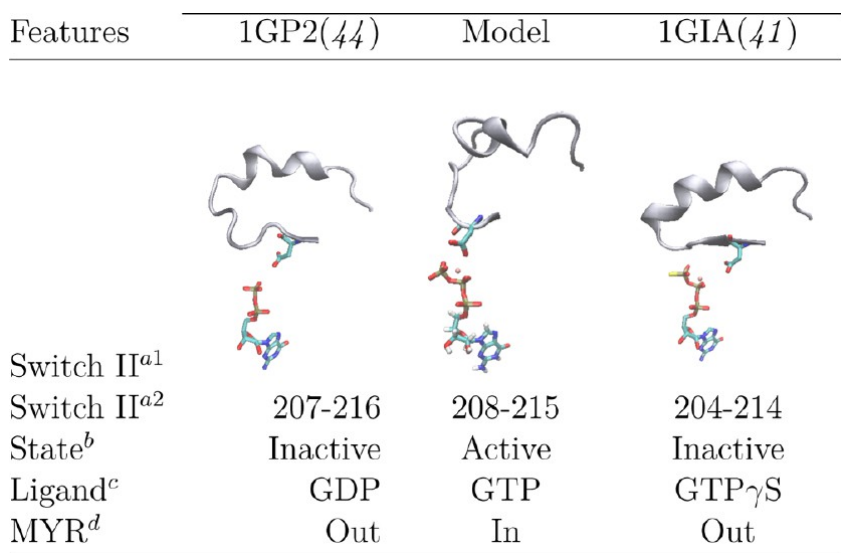

${ }^{a}$ Structures of $\mathrm{G} \alpha_{i 1}$ in different states specified via their PDB code. The middle column describes the presented model. (a1) Residues 200-219 are shown in the depiction of the switch II region together with ligands: $\mathrm{Mg}^{2+}$ and GTP, GTP $\gamma$ S or GDP. (a2) Shows the sequence of residues that are included in the helical parts of the switch II region. (b) Shows if the structure is able to interact with the down stream protein adenylyl cyclase type 5 . (c) Specifies which guanine nucleotide is interacting with the $\mathrm{G} \alpha_{i 1}$ subunit. (d) Shows the location of the myristoyl group (MYR) in comparison to the rest of the protein. If MYR is in, the N-terminal tail is interacting with the hydrophobic binding pocket. If MYR is out, the myristoyl group is not present in the system, and therefore the moiety is not interacting with the rest of the $\mathrm{G} \alpha_{i 1}$ subunit.

$\mathrm{G} \alpha_{i 1}^{\text {non }}-\mathrm{GDP}$ complex, in which the majority of the $\mathrm{N}$-terminus is resolved, suggests that the myristoyl group is located near the membrane and is not able to interact with the hydrophobic pocket. The $\mathrm{G} \alpha_{i 1}^{\text {non }}-\mathrm{GTP} \gamma \mathrm{S}$ structure is a conformation in which the entire $\mathrm{N}$-terminus is absent and could therefore be perceived as a conformation of active $\mathrm{G} \alpha_{i 1}$ with the myristoyl group not docked in the hydrophobic pocket but in a different hydrophobic region, such as the membrane. When $\mathrm{G} \alpha_{i 1}^{\text {non }}$ GTP $\gamma \mathrm{S}, \mathrm{G} \alpha_{i 1}^{\mathrm{myr}}-\mathrm{GTP}$, and $\mathrm{G} \alpha_{i 1}^{\mathrm{non}}-\mathrm{GDP}$ are compared, the location of the myristoyl also appears to affect other regions of the subunit than the hydrophobic binding pocket, such as the switch II region (Table 1).

The differences in the switch II region between the three states are evident. A unique feature of the solvated $\mathrm{G} \alpha \alpha_{i 1}^{\text {myr }}$ state, for instance, is the addition of two residues to the switch II region due to the downward shift of $\beta 2-\beta 3$ (Figure 7 ). This elongation of switch II leads to a stability enhancement of this domain because of its ability to interact with the $\alpha 3$ helix (Figure 3). The C-terminal region of the switch II region is structured via two or three helical turns that lead to a hydrophobic pocket for Trp211 (Figure 7 and Supporting Graph 1). The obtained structure of the $\mathrm{G} \alpha_{i 1}^{\mathrm{myr}}-\mathrm{GTP}$ switch II region is consistent with experimental data ${ }^{5}$ that show that Trp211 becomes located in a more hydrophobic environment when a myristoyl group is attached to the N-terminus of $\mathrm{G} \alpha_{i 1}$. Figure 7 depicts the conformation of switch II in both $\mathrm{G} \alpha_{i 1}^{\text {non }}-$ $\mathrm{GTP} \gamma \mathrm{S}$ and $\mathrm{G} \alpha_{i 1}^{\mathrm{myr}}-\mathrm{GTP}$. From a structural inspection it can be concluded that Trp211 in $\mathrm{G} \alpha_{i 1}^{\mathrm{myr}}-\mathrm{GTP}$ is mainly shielded from water by the switch II region itself and by $\alpha 3-\beta 5$. However, due to the conformation of switch II in the G $\alpha_{i 1}^{\text {non }}-\mathrm{GTP} \gamma \mathrm{S}$ X-ray structure, Trp211 appears to be solvent exposed, which is not in line with the hydrogen/deuterium exchange analysis performed by Preininger et al. ${ }^{5}$

Besides the structural changes of the Ras domain, the $\mathrm{AH}$ domain also adjusts upon myristoyl binding. Especially the region that is not part of the interaction site with the Ras domain is significantly altered compared to the initial conformation. The regions in which the largest change can be observed are in the $\alpha \mathrm{A}$ and the $\alpha \mathrm{B}$ helices (Figure 7c and Supporting Figure 4a). $\alpha \mathrm{B}$ 's initial conformation consists of four helical turns from Asp97 to Ala111 and two helical turns from Gly112 to Gly117. The final conformation of $\alpha \mathrm{B}$ includes a double helical turn from Asp97 to Ala104 and three helical turns from Arg105 to Ala113. Since the secondary structure around the Ras domain and the $\mathrm{AH}$ domain is marginally changed, the only driving force behind these alterations in $\alpha \mathrm{B}$ is the conformational change of the switch I region. During the classical MD simulation, the switch I region optimizes its interactions with the environment, which leads to an altered conformation that is able to get into close proximity of the $\mathrm{AH}$ domain. Residues Glu115 and Glu116 in helix $\alpha \mathrm{B}$ are biased toward the highly charged region around the GTP binding site that is located more on the Ras domain in the $\mathrm{G} \alpha_{i 1}^{\mathrm{my}}-\mathrm{GTP}$ complex than in the G $\alpha_{i 1}^{\text {non }}-\mathrm{GTP}$ structure (Figure 8). Furthermore, the switch I region is able to come closer to $\alpha \mathrm{B}$ because of this conformational change. These two characteristics lead to a kinked helix that includes an unstructured region between Ala114 and Met119 that connects the $\alpha \mathrm{B}$ helix to $\alpha \mathrm{C}$. This unstructured region contains Glu115 and Glu116 that can interact with switch I through interactions with Thr181 and the backbone of Lys180. Because of its structural changes, the $\alpha \mathrm{B}$ helix modifies the surface and electrostatics of the protein and is able to interact with the switch I region.

Electrostatic Properties and Protein-Protein Interaction Site. Besides the structural changes, also the electrostatic properties around the switch II region are significantly different when myristoylated and nonmyristoylated active $\mathrm{G} \alpha_{i 1}$ states are compared (Figure 8). First, the positively charged region around the GTP binding site is more narrowly localized on the Ras domain in the soluble $\mathrm{G} \alpha_{i 1}^{\mathrm{myr}}$ conformation compared to the $\mathrm{G} \alpha_{i 1}^{\text {non }}-\mathrm{GTP} \gamma \mathrm{S}$ structure. Second, the area 

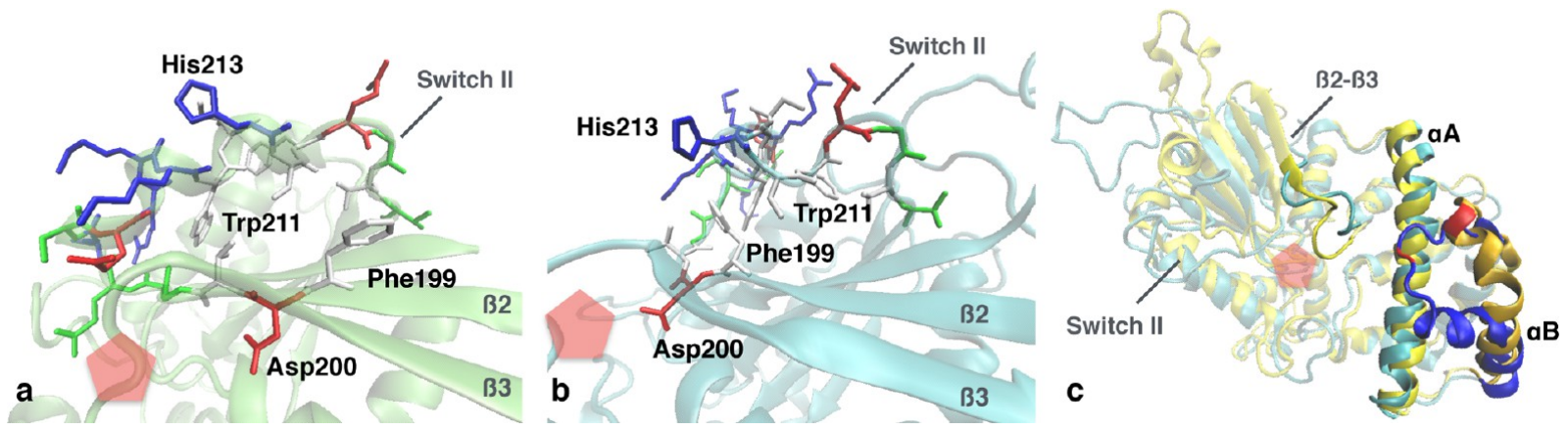

Figure 7. Comparison of the switch II region of $\mathrm{G} \alpha_{i 1}^{\mathrm{myr}}-\mathrm{GTP}$ and $\mathrm{G} \alpha_{i 1}^{\mathrm{non}}-\mathrm{GTP} \gamma \mathrm{S}$ together with the change in conformation of the AH domain. (a) Detailed description of the switch II region of the 1GIA PDB structure. Nonpolar residues are depicted in white, polar residues in green, positively charged residues in blue and negatively charged residues in red. The nucleotide binding site is shown via the red pentagon. (b) Detailed description of the switch II region of the solvated $\mathrm{G} \alpha_{i 1}^{\mathrm{myr}}-\mathrm{GTP}$ structure. The color scheme for the residues and nucleotide interacting site is the same as in the (a) image. (c) Conformational changes in the $\mathrm{AH}$ domain when comparing the initial conformation (yellow) to a conformation of the protein after $\sim 1.8 \mu \mathrm{s}$ (blue). The red regions on the helices depict the location of Glu115 and Glu116. The red pentagon represents the location of the GTP binding site.

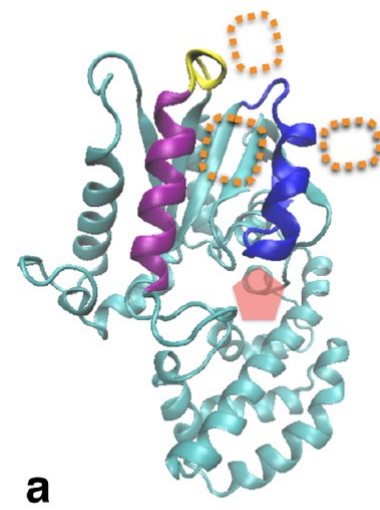

b
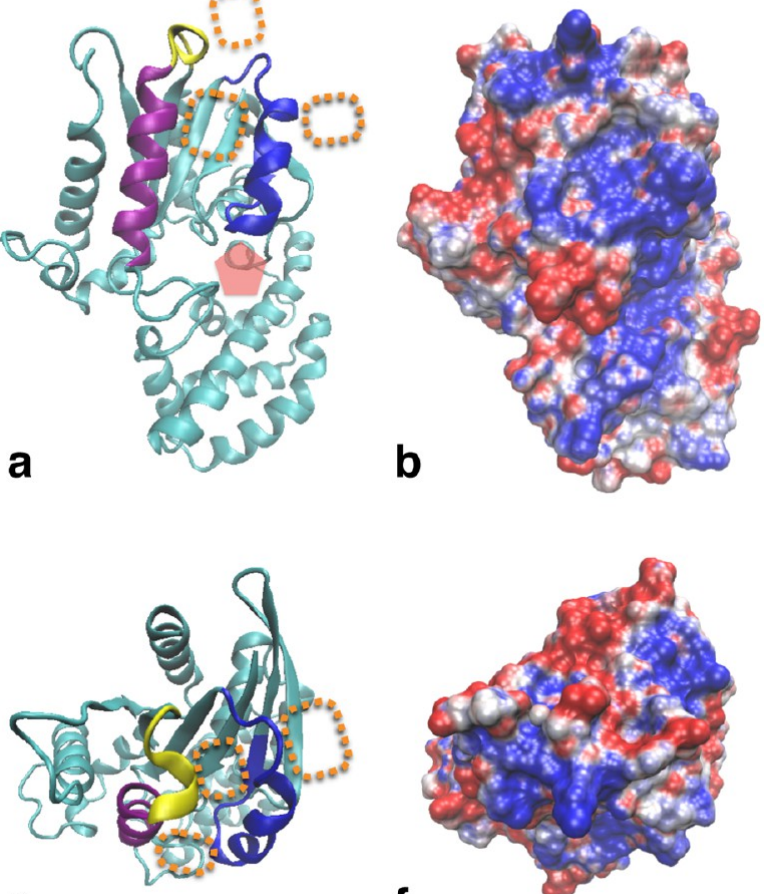

e

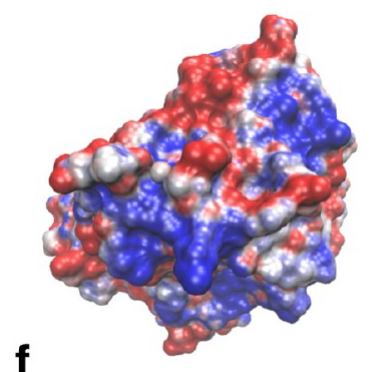

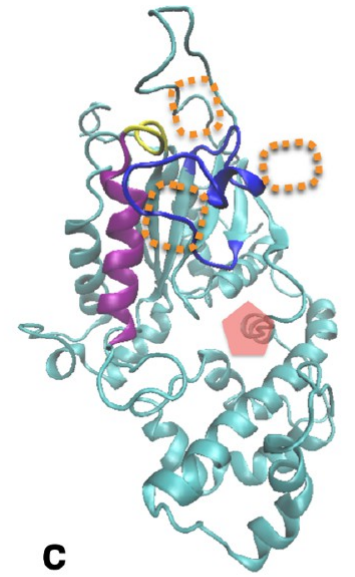

d
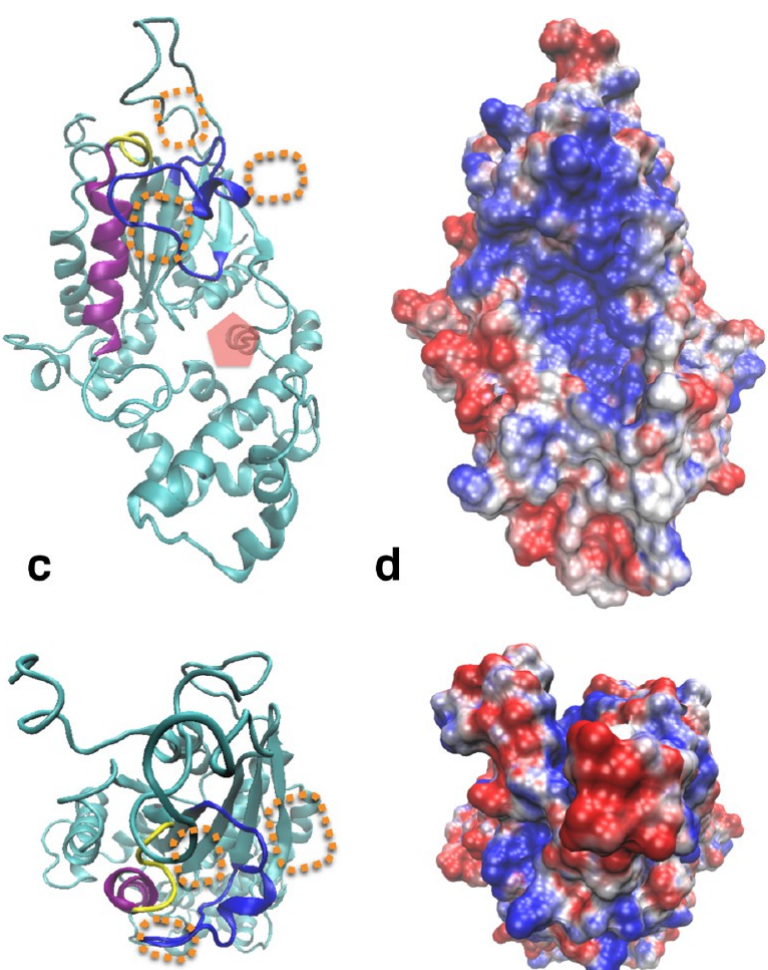

g

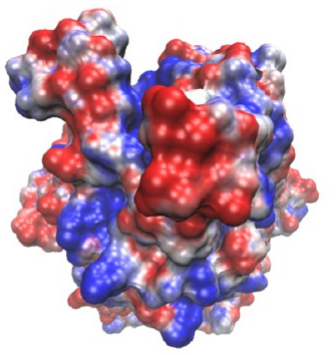

h

Figure 8. Comparison of the $\mathrm{G} \alpha_{i 1}^{\text {non }}-\mathrm{GTP} \gamma \mathrm{S}$ complex and $\mathrm{G} \alpha_{i 1}^{\mathrm{myr}}-\mathrm{GTP}$. Front (a) and top (e) view of the G $\alpha_{i 1}^{\text {non }} \mathrm{X}$-ray structure (PDB code: $1 \mathrm{GIA}$ ) in complex with GTP $\gamma \mathrm{S}$, a GTP analog. The pentagon indicates the location of the GTP $\gamma \mathrm{S}$ molecule and the orange dotted rectangles represent the regions at which AC5 binds to $\mathrm{G} \alpha_{s}$ in the X-ray structure (PDB code: 1CJK). The blue region shows the switch II region, the purple helix is $\alpha 3$ and the yellow part of the protein is $\alpha 3-\beta 5$. (b, f) Electrostatic surface maps the front (b) and top (f) view of a G $\alpha_{i 1}^{\text {non }} \mathrm{X}$-ray structure (PDB code: $1 \mathrm{GIA}$ ) interacting with GTP $\gamma$ S. Three colors are employed: blue for positive values of the electrostatic potential, white for uncharged regions and red for negatively charged areas. The surface of the $\mathrm{G} \alpha_{i 1}$ protein is contoured from -3 (red) to +3 (blue) kT/e based on the electrostatic potential present at the solvent accessible surface. $(\mathrm{c}, \mathrm{g})$ Front $(\mathrm{c})$ and top $(\mathrm{g})$ view of the $\mathrm{G} \alpha_{i 1}^{\text {myr }}$ structure after about $1.8 \mu \mathrm{s}$. The coloring scheme is the same as in image (a). ( $\mathrm{d}, \mathrm{h}$ ) Electrostatic surface map of the front (d) and top (h) view of the $\mathrm{G} \alpha_{i 1}^{\mathrm{myr}}$ structure after about $1.8 \mu \mathrm{s}$ of classical MD. The color coding is the same as in image (b).

around the $\alpha 3$ helix is less negatively charged in the $\mathrm{G} \alpha_{i 1}^{\mathrm{myr}}-$ GTP complex with respect to the $\mathrm{G} \alpha_{i 1}^{\text {non }}-\mathrm{GTP} \gamma \mathrm{S}$ conformation. Third, the negatively charged patch created by the $\mathrm{N}$-terminus near the C-terminal side of switch II (Figure $8 \mathrm{e}-\mathrm{h}$ ), is not present in $\mathrm{G} \alpha_{i 1}^{\text {non }}-\mathrm{GTP} \gamma \mathrm{S}$ as this region is not resolved in the $\mathrm{X}$-ray structure.
Commonly, G $\alpha_{i 1}$ 's switch II region, close to the GTP binding site, is proposed to be the main interaction site for down stream proteins like adenylyl cyclase. In the case of AC, two pseudo symmetrical sites are present on the protein of which one, the one located on the $\mathrm{C} 2$ domain, is known to interact with the activated form of $\mathrm{G} \alpha_{s}$ via its switch II region (Supporting Figure 9). Gel-filtration and activity experiments have shown that the 
interaction of AC5 with both G proteins is mainly non competitive and that $\mathrm{G} \alpha_{s}$ can only form a complex with $\mathrm{C} 2$ while $\mathrm{G} \alpha_{i 1}^{\mathrm{myr}}$ complexes solely with the $\mathrm{C} 1$ domain (Supporting Figure 9). ${ }^{5,16}$ Hence, the $\mathrm{C} 1$ domain is proposed to interact with $\mathrm{G} \alpha_{i 1}^{\mathrm{myr}}-\mathrm{GTP}$ 's switch II region as X-ray structures of both activated $\mathrm{G} \alpha_{s}$ and $\mathrm{G} \alpha_{i 1}^{\text {non }}$ are structurally similar (Figure 8a,e). However, upon solvation, the conformation of $\mathrm{G} \alpha_{i 1}^{\text {myr }}$ significantly changes compared to the nonmyristoylated X-ray structure, including the switch II region. Therefore, a similar interaction site on $\mathrm{G} \alpha_{i 1}^{\mathrm{myr}}-\mathrm{GTP}$ for $\mathrm{C} 1$ as the one on $\mathrm{G} \alpha_{s}$ :GTP for the C2 domain, located between $\alpha 3$ and switch II, appears to become less favorable as both switch II and the N-terminus are shielding this region (Supporting Figure 9 and Figure 8c,g). This change in conformation could indicate that a different interaction site for AC type 5 exists on the soluble $\mathrm{G} \alpha_{i 1}^{\mathrm{myr}}-\mathrm{GTP}$ structure. Such a view is also supported by the fact that AC type 5 is only inhibited by $\mathrm{G} \alpha_{i 1}^{\text {myr }}-\mathrm{GTP}$ (Table 1 ), as $\mathrm{G} \alpha_{i 1}^{\text {non }}-\mathrm{GTP}$ loses its inhibiting function and is unable to interact with AC5. ${ }^{17}$ Furthermore, $\mathrm{G} \alpha_{i 1}^{\mathrm{myr}}-\mathrm{GTP}$ is even able to inhibit AC5 when only the catalytic domains $(\mathrm{C} 1-\mathrm{C} 2)$ are taken into account, which suggests that $\mathrm{G} \alpha_{i 1}^{\mathrm{myr}}-\mathrm{GTP}$ is able to interact with AC5 in the cytosol. ${ }^{16}$ Therefore, we propose here that the soluble form of $\mathrm{G} \alpha_{i 1}^{\mathrm{myr}}-\mathrm{GTP}$ could be a good candidate for AC5 binding.

Conclusions. In the absence of myristoylation of the $\mathrm{N}$ terminus, experimental studies have been able to elucidate the structure of active and inactive $\mathrm{G} \alpha_{i 1}^{\text {non }}$. However, this $\mathrm{N}$-terminal modification is present under physiological conditions and is known to affect the subunit's conformation and function in the protein's GTP-bound state. In the case of inactive $\mathrm{G}_{i 1}^{\mathrm{myr}}-\mathrm{GDP}$, the complex is membrane bound, and the myristoyl group interacts with the membrane and is not located close to the Ras domain of the $\mathrm{G} \alpha_{i 1}$ subunit. However, the conformation of an active myristoylated $\alpha$ subunit can be affected by the myristoyl group as the $\alpha$ subunit can relocate to the cytosol due to the dissociation from the $\beta \gamma$ dimer and the depalmitoylation of the $\mathrm{N}$-terminus, therefore providing the opportunity for the myristoyl group to interact with the $\alpha$ subunit. The performed $2 \mu \mathrm{s}$ simulation of the conformation of active $\mathrm{G} \alpha_{i 1}^{\mathrm{myr}}$ shows that a solvated G $\alpha_{i 1}-$ GTP's structure is able to adopt a conformation in which the myristoyl moiety is embedded in a hydrophobic binding pocket on the protein itself. The presented conformation of the $\mathrm{G} \alpha_{i 1}^{\mathrm{myr}}-\mathrm{GTP}$ complex provides a possible explanation for the disagreement between the $\mathrm{G} \alpha_{i 1}^{\text {non }}-$ $\mathrm{GTP} \gamma \mathrm{S}$ X-ray structure and other experiments on active $\mathrm{G} \alpha_{i 1}^{\mathrm{myr}}$, such as fluorescence and hydrogen/deuterium exchange experiments.

The structural rearrangements in solvated $\mathrm{G} \alpha_{i 1}^{\mathrm{myr}}-\mathrm{GTP}$ present a new view on $\mathrm{G} \alpha_{i 1}$,s possibilities for protein-protein interactions as for its interaction with AC5, for instance. AC5 is present in high concentrations in the brain and heart, which has been associated with congestive heart failure and pain perception. ${ }^{45,46}$ Inhibitors of AC5 are therefore proposed as possible drug target for treatment of heart failure and analgesics. ${ }^{45}$ Hence, obtaining a better view on how these proteins interact could be important for the development of AC5 inhibitors. Additionally, the presence of two active $\mathrm{G} \alpha_{i 1}$ conformations, membrane-bound and solvated, constitutes a significant difference between $\mathrm{G} \alpha_{i 1}$ and $\mathrm{G} \alpha_{s}$ as it is suggested that $\mathrm{G} \alpha_{s}$ only adopts one conformation of the Ras and the alpha helical domain with a structured or unstructured $\mathrm{N}$-terminus in the active state, depending on its location in the cell. Consequently, although $\mathrm{G} \alpha_{s}$ is proposed to lose its structure of the N-terminus upon activation and depalmitoylation, the overall conformation of the protein could remain the same in the solvated state compared to the membrane-bound conformation as $\mathrm{G} \alpha_{s}$, without its $\mathrm{N}$-terminus, is still able to interact with AC5. ${ }^{18}$ This striking difference between $\mathrm{G} \alpha_{i 1}$ and $\mathrm{G} \alpha_{s}$ could provide an alternative view of the function of $\mathrm{G}$ proteins in their active conformation. Moreover this study shows that lipid interactions can be crucial for the conformation of proteins and that through lipidation the function of structurally highly similar proteins can be altered and regulated.

\section{ASSOCIATED CONTENT}

\section{S Supporting Information}

The Supporting Information is available free of charge on the ACS Publications website at DOI: 10.1021/acs.biochem.6b00388.

Nine figures, a table, and two graphs. More information on the myristoyl binding site and the GTP binding site as an addition to the Results and Discussion section (PDF)

\section{AUTHOR INFORMATION}

\section{Corresponding Author}

*E-mail: ursula.roethlisberger@epfl.ch.

ORCID

Siri C. van Keulen: 0000-0001-6995-8389

\section{Author Contributions}

S.C.v.K. ran simulations and prepared the setup for the simulations and alignments and performed the analysis and wrote the text. U.R. supervised the project and also wrote the text.

\section{Funding}

This work was supported by the Swiss National Science Foundation Grant No. 200020_146645 and computer time on the DENEB SCITAS cluster.

\section{Notes}

The authors declare no competing financial interest.

\section{ACKNOWLEDGMENTS}

The authors thank Dr. Pietro Vidossich for help with the $\mathrm{Mg}^{2+}$, $\mathrm{K}^{+}$, and $\mathrm{Cl}^{-}$force field parameters. The authors thank Dr. Murat Kiliç for helping with preparing the response to the reviewers.

\section{REFERENCES}

(1) Dror, R. O., Mildorf, T. J., Hilger, D., Manglik, A., Borhani, D. W., Arlow, D. H., Philippsen, A., Villanueva, N., Yang, Z., Lerch, M. T., Hubbell, W. L., Kobilka, B. K., Sunahara, R. K., and Shaw, D. E. (2015) Structural basis for nucleotide exchange in heterotrimeric $G$ proteins. Science 348, 1361-1365.

(2) Louet, M., Martinez, J., and Floquet, N. (2012) GDP release preferentially occurs on the phosphate side in heterotrimeric Gproteins. PLoS Comput. Biol. 8, e1002595.

(3) Schröter, G., Mann, D., Kötting, C., and Gerwert, K. (2015) Integration of Fourier Transform Infrared Spectroscopy, Fluorescence Spectroscopy, Steady-state Kinetics and Molecular Dynamics Simulations of G $\alpha$ il Distinguishes between the GTP Hydrolysis and GDP Release Mechanism. J. Biol. Chem. 290, 17085-17095.

(4) Galés, C., Van Durm, J. J., Schaak, S., Pontier, S., Percherancier, Y., Audet, M., Paris, H., and Bouvier, M. (2006) Probing the activation-promoted structural rearrangements in preassembled receptor-G protein complexes. Nat. Struct. Mol. Biol. 13, 778-786.

(5) Preininger, A. M., Kaya, A. I., Gilbert, J. A., III, Busenlehner, L. S., Armstrong, R. N., and Hamm, H. E. (2012) Myristoylation exerts 
direct and allosteric effects on $\mathrm{G} \alpha$ conformation and dynamics in solution. Biochemistry 51, 1911-1924.

(6) Liu, Y., Kahn, R. A., and Prestegard, J. H. (2009) Structure and membrane interaction of myristoylated ARF1. Structure 17, 79-87.

(7) Liu, Y., Kahn, R. A., and Prestegard, J. H. (2010) Dynamic structure of membrane-anchored Arf [bull] GTP. Nat. Struct. Mol. Biol. 17, 876-881.

(8) Behnia, R. and Munro, S. (2005) Organelle identity and the signposts for membrane traffic. Nature 438, 597-604.

(9) Chen, C. A., and Manning, D. R. (2001) Regulation of G proteins by covalent modification. Oncogene 20, 1643-1652.

(10) Cabrera-Vera, T. M., Vanhauwe, J., Thomas, T. O., Medkova, M., Preininger, A., Mazzoni, M. R., and Hamm, H. E. (2003) Insights into $\mathrm{G}$ protein structure, function, and regulation. Endocr. Rev. 24, 765-781.

(11) Wright, M. H., Heal, W. P., Mann, D. J., and Tate, E. W. (2010) Protein myristoylation in health and disease. Journal of chemical biology 3, 19-35.

(12) Loisel, T. P., Ansanay, H., Adam, L., Marullo, S., Seifert, R., Lagacé, M., and Bouvier, M. (1999) Activation of the $\beta 2$-adrenergic receptor-G $\alpha$ s complex leads to rapid depalmitoylation and inhibition of repalmitoylation of both the receptor and G $\alpha$ s. J. Biol. Chem. 274, 31014-31019.

(13) Wedegaertner, P. B., and Bourne, H. R. (1994) Activation and depalmitoylation of $\mathrm{G} s \alpha$. Cell 77, 1063-1070.

(14) Degtyarev, M. Y., Spiegel, A. M., and Jones, T. (1994) Palmitoylation of a $\mathrm{G}$ protein alpha i subunit requires membrane localization not myristoylation. J. Biol. Chem. 269, 30898-30903.

(15) Preininger, A. M., Van Eps, N., Yu, N.-J., Medkova, M., Hubbell, W. L., and Hamm, H. E. (2003) The myristoylated amino terminus of G $\alpha$ il plays a critical role in the structure and function of G $\alpha$ il subunits in solution. Biochemistry 42, 7931-7941.

(16) Dessauer, C. W., Tesmer, J. J., Sprang, S. R., and Gilman, A. G. (1998) Identification of a Gi $\alpha$ binding site on type V adenylyl cyclase. J. Biol. Chem. 273, 25831-25839.

(17) Taussig, R., Iniguez-Lluhi, J. A., and Gilman, A. G. (1993) Inhibition of adenylyl cyclase by Gi alpha. Science 261, 218-221.

(18) Tesmer, J. J., Sunahara, R. K., Johnson, R. A., Gosselin, G., Gilman, A. G., and Sprang, S. R. (1999) Two-metal-ion catalysis in adenylyl cyclase. Science 285, 756-760.

(19) Berman, H. M., Westbrook, J., Feng, Z., Gilliland, G., Bhat, T., Weissig, H., Shindyalov, I. N., and Bourne, P. E. (2000) The protein data bank. Nucleic Acids Res. 28, 235-242.

(20) Eswar, N., Webb, B., Marti-Renom, M. A., Madhusudhan, M., Eramian, D., Shen, M.-y., Pieper, U., and Sali, A. (2006) Comparative protein structure modeling using Modeller. Curr. Protocols Bioinformatics, 5.6.1-5.6.30.

(21) Martí-Renom, M. A., Stuart, A. C., Fiser, A., Sánchez, R., Melo, F., and Šali, A. (2000) Comparative protein structure modeling of genes and genomes. Annu. Rev. Biophys. Biomol. Struct. 29, 291-325.

(22) Kaya, A. I., Lokits, A. D., Gilbert, J. A., Iverson, T. M., Meiler, J., and Hamm, H. E. (2014) A conserved phenylalanine as a relay between the $\alpha 5$ helix and the GDP binding region of heterotrimeric Gi protein $\alpha$ subunit. J. Biol. Chem. 289, 24475-24487.

(23) Raw, A. S., Coleman, D. E., Gilman, A. G., and Sprang, S. R. (1997) Structural and Biochemical Characterization of the GTP $\gamma$ S-, GDP Pi-, and GDP-Bound Forms of a GTPase-Deficient Gly $42 \rightarrow$ Val Mutant of Gi $\alpha 1$. Biochemistry 36, 15660-15669.

(24) Pasqualato, S., Renault, L., and Cherfils, J. (2002) Arf, Arl, Arp and Sar proteins: a family of GTP-binding proteins with a structural device for 'front-back'communication. EMBO Rep. 3, 1035-1041.

(25) Hornak, V., Abel, R., Okur, A., Strockbine, B., Roitberg, A., and Simmerling, C. (2006) Comparison of multiple Amber force fields and development of improved protein backbone parameters. Proteins: Struct., Funct., Genet. 65, 712-725.

(26) Jorgensen, W. L., Chandrasekhar, J., Madura, J. D., Impey, R. W., and Klein, M. L. (1983) Comparison of simple potential functions for simulating liquid water. J. Chem. Phys. 79, 926-935.
(27) Bekker, H., Berendsen, H., Dijkstra, E., Achterop, S., Van Drunen, R., Van der Spoel, D., Sijbers, A., Keegstra, H., Reitsma, B., and Renardus, M. (1993) Gromacs: A parallel computer for molecular dynamics simulations. Phys. Comput. 92, 252-256.

(28) Hess, B., Kutzner, C., Van Der Spoel, D., and Lindahl, E. (2008) GROMACS 4: algorithms for highly efficient, load-balanced, and scalable molecular simulation. J. Chem. Theory Comput. 4, 435-447.

(29) Meagher, K. L., Redman, L. T., and Carlson, H. A. (2003) Development of polyphosphate parameters for use with the AMBER force field. J. Comput. Chem. 24, 1016-1025.

(30) Joung, I. S., and Cheatham, T. E., III (2008) Determination of alkali and halide monovalent ion parameters for use in explicitly solvated biomolecular simulations. J. Phys. Chem. B 112, 9020-9041.

(31) Allnér, O., Nilsson, L., and Villa, A. (2012) Magnesium ionwater coordination and exchange in biomolecular simulations. J. Chem. Theory Comput. 8, 1493-1502.

(32) Frisch, M. J. et al. Gaussian 09, Revision D.01. Gaussian Inc.: Wallingford, CT, 2009.

(33) Case, D., Berryman, J., Betz, R., Cerutti, D., Cheatham, T., III, Darden, T., Duke, R., Giese, T., Gohlke, H., and Goetz, A. AMBER 2015; University of California: San Francisco, CA, 2015.

(34) Baker, N. A., Sept, D., Joseph, S., Holst, M. J., and McCammon, J. A. (2001) Electrostatics of nanosystems: application to microtubules and the ribosome. Proc. Natl. Acad. Sci. U. S. A. 98, 10037-10041.

(35) Unni, S., Huang, Y., Hanson, R. M., Tobias, M., Krishnan, S., Li, W. W., Nielsen, J. E., and Baker, N. A. (2011) Web servers and services for electrostatics calculations with APBS and PDB2PQR. J. Comput. Chem. 32, 1488-1491.

(36) Dolinsky, T. J., Czodrowski, P., Li, H., Nielsen, J. E., Jensen, J. H., Klebe, G., and Baker, N. A. (2007) PDB2PQR: expanding and upgrading automated preparation of biomolecular structures for molecular simulations. Nucleic Acids Res. 35, W522-W525.

(37) Shatsky, M., Nussinov, R., and Wolfson, H. J. (2004) A method for simultaneous alignment of multiple protein structures. Proteins: Struct., Funct., Genet. 56, 143-156.

(38) Humphrey, W., Dalke, A., and Schulten, K. (1996) VMD: visual molecular dynamics. J. Mol. Graphics 14, 33-38.

(39) UniProt Consortium. (2015) UniProt: a hub for protein information. Nucleic Acids Res. 43, D204-D212.

(40) Sunahara, R. K., Tesmer, J. J., Gilman, A. G., and Sprang, S. R. (1997) Crystal structure of the adenylyl cyclase activator Gs $\alpha$. Science 278, 1943-1947.

(41) Coleman, D. E., Berghuis, A. M., Lee, E., Linder, M. E., Gilman, A. G., and Sprang, S. R. (1994) Structures of active conformations of Gi alpha 1 and the mechanism of GTP hydrolysis. Science 265, 14051412 .

(42) Linder, M. E., Pang, I.-H., Duronio, R., Gordon, J. I., Sternweis, P., and Gilman, A. (1991) Lipid modifications of $G$ protein subunits. Myristoylation of Go alpha increases its affinity for beta gamma. J. Biol. Chem. 266, 4654-4659.

(43) Galbiati, F., Guzzi, F., Magee, A., Milligan, G., and Parenti, M. (1996) Chemical inhibition of myristoylation of the G-protein Gil alpha by 2-hydroxymyristate does not interfere with its palmitoylation or membrane association. Evidence that palmitoylation, but not myristoylation, regulates membrane attachment. Biochem. J. 313, 717720.

(44) Wall, M. A., Coleman, D. E., Lee, E., Iñiguez-Lluhi, J. A., Posner, B. A., Gilman, A. G., and Sprang, S. R. (1995) The structure of the G protein heterotrimer $\mathrm{G}$ i $\alpha 1 \beta 1 \gamma 2$. Cell 83, 1047-1058.

(45) Pierre, S., Eschenhagen, T., Geisslinger, G., and Scholich, K. (2009) Capturing adenylyl cyclases as potential drug targets. Nat. Rev. Drug Discovery 8, 321-335.

(46) Sadana, R., and Dessauer, C. W. (2009) Physiological roles for $\mathrm{G}$ protein-regulated adenylyl cyclase isoforms: insights from knockout and overexpression studies. Neurosignals 17, 5-22. 